\title{
WHEN OFF TARGET EFFECTS ARE ON TARGET: THE ROLE OF SPIRONOLACTONE IN PATIENTS WITH COVID-19
}

Christopher Wilcox, MD PhD ${ }^{1}$, Bertram Pitt, $\mathrm{MD}^{2}$

${ }^{1}$ Division of Nephrology and Hypertension Center, Georgetown University, Washington DC 20007

${ }^{2}$ Division of Cardiology, University of Michigan, Ann Arbor, MI 48109

Short Title: Spironolactone off target effects

Address for correspondence:

Christopher Wilcox, MD, $\mathrm{PhD}$

Georgetown University Department of Nephrology and Hypertension

3800 Reservoir Road, NW

PHC F6003

Washington, DC 20007

Fax: 877-625-1483

Email: wilcoxch@georgetown.edu 


\begin{abstract}
Aims: Spironolactone is a steroidal mineralocoricosteroid receptor antagonist (MRA) used for treatment of resistant hypertension, heart failure and edema. It exerts class specific adverse effects that are shared by other MRAs. Additionally, it exerts unique "off target" steroidal effects that include gynecomastia, impotence and loss of libido in males and menstrual irregularity in females. Together, these have led to a poor tolerability and limited use despite positive results in many randomized, controlled clinical trials. We review the off-target effects of spironolactone that may summate with its MRA action to provide an advantageous profile for prevention or treatment of patients with COVID-19. Methods: Literature review using PubMed Central. Results: The blockade by spironolactone of the androgen receptor should diminish the expression of transmembrane protease serine 2 (TMPRSS2) that has an androgen promoter while its MRA action should enhance the expression of protease nexin1 (PN1) that inhibits furin and plasmin. TMPRSS2, furin and plasmin cooperated to process the SARS-CoV-2 spike protein to increase its high affinity binding to the angiotensin converting enzyme 2 (ACE2) and thereby promote viral cell entry. Its actions as an MRA may reduce inflammation and preserve pulmonary, cardiac and vascular functions. Its anti-plasmin action may combat hemostatic dysfunction. Conclusion: The hypothesis that the off-target effects of spironolactone summate with its MRA actions to provide special benefits for COVID-19 is worthy of direct investigation and clinical trial.
\end{abstract}

Keywords: mineralocorticosteroid receptor antagonist (MRA); angiotensin converting enzyme 2 (ACE2); SARS-CoV-2; transmembrane protease receptor serine 2; furin; plasmin 
Introduction: Most "off-target" drug effects are detrimental. In this hypothesis review, we consider the off-target effects of spironolactone that may become an advantage if used to prevent or limit the severity of the SARS-CoV-2 virus infection. We review the steroidal "off-target" and the mineralocorticoid receptor antagonist (MRA) "on-target" actions of spironolactone and their potential impact on COVID-19 disease.

Spironolactone was used initially as a diuretic but became established as a drug of choice to treat drug resistant hypertension and has earned a class 1 indication for the treatment of heart failure with reduced ejection fraction (HFrEF) in both US and European guidelines based on the results of the Optimum Treatment for Drug Resistant Hypertension (PATHWAY-2) (1), Randomized Aldactone Evaluation Study (RALES) (2), Eplerenone Post-Acute Myocardial Infarction Study (EPHESUS) (3) and Eplerenone in Patients with Systolic Heart Failure (EMPHASIS-HF) (4) trials. Despite these recommendations, the use of spironolactone has been lessened because of reduced tolerability that derives from a combination of adverse MRA class effects and off-target effects that are specific for spironolactone.

Recent interest has been expressed in a novel use for spironolactone to prevent, or moderate, the symptoms, of COVID-19 first by others (5-7) and by us (8). Moreover, several clinical trials of spironolactone in COVID-19 are planned, or have begun recruitment: Losartan and Spironolactone Treatment for ICU patients with COVID-19 suffering from ARDS (COVIDANCE: NTC 04643691; Bromhexine and Spironolactone for CoronavirUs Infection Requiring HospiTalization (BISCUIT): NTC04345887; Spironolactone in COVID-19 Induced ARDS: NTC04345887). Spironolactone exerts both MRA and the anti-androgen actions that may contribute to protection from COVID-19 (9). Indeed, a small observational trial has reported a significantly reduced rate of admission of COVID-19 infected men taking anti-androgen 
medications (primarily 5 - $\alpha$-reductase inhibitors for prostate cancer) to the intensive care unit (10) while a population-based retrospective study among 4532 male patients from Italy reported a significant 4-fold protection among prostate cancer patients receiving androgen-depriving therapies (11). We present a hypothesis for the relative potential efficacy of spironolactone exerted by its class vs off-target actions, and the implications for its use to prevent or lessen the severity of COVID-19 infection.

Class effects of spironolactone: The major adverse class effect is hyperkalemia that can be a serious, and potentially fatal, complication. It is reported in $19 \%$ of patients over the first year of spironolactone therapy and leads to withdrawal of spironolactone in one half of these (12) but is rarely a problem in patients with normal renal function. Hyperkalemia can often be managed with patiromer or zirconium sodium. MRAs also can cause a hemodynamic reduction in the glomerular filtration rate (GFR) but over the long term they may slow the decline in GFR.

Off-target effects of spironolactone: The major off-target effects are breast pain, gynecomastia, impotence and loss of libido in males and menstrual irregularities in females. Spironolactone also can release adrenocorticotropic hormone (ACTH) and raise plasma cortisol (13). The steroidal off-target effects of spironolactone include anti-androgen, pro-progestin and pro-glucocorticoid actions, although the former predominate (14). These are time- and dosedependent. Anti-androgen effects are encountered in more than half of the patients receiving spironolactone at a high dosage of $100 \mathrm{mg}$ for more than three months (15). The off-target actions have motivated the development of MRAs with lesser effects on serum potassium or more specific binding to the MR and thereby absence of off-target actions.

Differences between MRAs: Eplerenone is a second generation steroidal MRA that lacks the off-target effects of spironolactone $(14,16,17)$. Third generation non-steroidal MRAs 
under development include finerenone, esaxerenone, aparenone and KBP-5074. These lack steroidal off-target effects and may also cause less hyperkalemia (18).

Mechanisms of the off-target effects of spironolactone: High concentrations of spironolactone, but not eplerenone, can interrupt steroidogenesis (19). Spironolactone may increase plasma progesterone (20) that has anti-androgenic activity in men. Despite these reports, even 10 months of administration of $200 \mathrm{mg}$ daily of spironolactone that induced gynecomastia in $62 \%$ of the male recipients, did not change plasma levels of testosterone, estradiol or progesterone (21). On balance, it is probable that the anti-androgenic activity of spironolactone is not due primarily to inhibition of testosterone generation.

There is stronger evidence that spironolactone inhibits the androgen receptor (AR). Thus, spironolactone and its metabolite canreonate inhibit the binding of radio- labelled 5-alphadihydrotestosterone to the cytosolic and nuclear AR in the prostate gland of the rat, where the affinity of spironolactone is 5-fold higher than canreonate (22). Inhibition of the AR by spironolactone is used to reduce androgenic effects in women with polycystic ovarian syndrome and hirsutism.

Spironolactone possesses only weak progesterogenic and anti-estrogenic activity but can regulate the subcellular distribution of estrogen receptors that could underlie its effects on intramenstrual bleeding and can inhibit 17-beta-hydrosteroid dehydrogenase type 2 that can increase estradiol (23).

\section{Circulating levels of renin, angiotensin II (Ang II) and aldosterone in COVID-19}

infected subjects: Presently lacking are serial measurements of the levels of renin, Ang II or aldosterone in patients infected with COVID-19 to provide additional rationale for use of renin- 
angiotensin-aldosterone system inhibitors (RAASi). However, a recent study of patients infected with SARS-CoV-2 reported a $45 \%$ incidence of hypokalemia with increased urinary potassium and systemic alkalosis, suggesting hyperaldosteronism (24).

A study of COVID-19 infected patients hospitalized with pneumonia reported a 3-fold increase in circulating levels of Ang II (25) while a similar study reported a 3-fold increase in circulating levels of renin and aldosterone in those severely affected (26). In contrast, studies in less severely affected patients in the US reported normal values for plasma Ang II and serum aldosterone (27). Apparently, the levels of RAAS components can be increased substantially during severe COVID-19 infection. Of interest, plasma levels of cortisol and ACTH were numerically (but not significantly) reduced in severely compromised, compared to mild/moderately compromised, patients with COVID-19 (26). Thus, the expected response of activation of the hypothalamic-pituitary-adrenal response by the stress of a severe illness may be suboptimal in COVID-19 infected patients. A metanalysis of dexamethasone use in COVID-19 patients with ARDS concluded that high dose steroids reduce mortality by $\sim 30 \%$ (28). However, it is not clear whether a modest increase in plasma cortisol, as reported in some studies with spironolactone, could be beneficial but is a potential component of its off-target effects, although clearly not a substitute for the use of high dose glucocorticosteroids in those developing ARDS.

Pharmacokinetic considerations: Spironolactone has a short half-life of less than 9 hours. It is metabolized principally to canrenone with a longer half-life of up to 58 hours. Adverse anti-androgen effects are less frequent with potassium canreonate than with spironolactone (29). Spironolactone, but not canreonate, downregulates hepatic ARs and upregulates estrogen receptors resulting in a $~ 10$-fold greater anti-androgen receptor activity than canrenone (30). Thus, the steroidal anti-androgen effects of spironolactone are mainly a 
property of the drug itself rather than its metabolite, yet both spironolactone and canrenone are potent inhibitors of the MRA.

Dose-dependent and metabolite effects of spironolactone: The Anglo-Scandinavian Cardiac Outcomes Trial-Blood Pressure Lowering Arm trial (ASCOT-BPLA) randomized participants to a mean daily dose of spironolactone of $25 \mathrm{mg}$ for 2.9 years. There was a significant fall in systolic blood pressure and a $6 \%$ rate of gynecomastia or breast discomfort in men (31) demonstrating a modest frequency of anti-androgen effects with relatively low doses of spironolactone even over several years. The anti-hypertensive efficacy of spironolactone increased from daily doses of $25 \mathrm{mg}$ to 75 to $100 \mathrm{mg}$, but there was no additional effect above $150 \mathrm{mg}$. Gynecomastia developed in $7 \%$ of men receiving daily doses of $50 \mathrm{mg}$ or less, but in $52 \%$ of those receiving $150 \mathrm{mg}$ or higher (32). Gynecomastia developed in $30 \%$ of normal men given spironolactone at $100 \mathrm{mg}$ daily for 10 months but in $62 \%$ of those given $200 \mathrm{mg}$ daily. Thus, both the anti-hypertensive and anti-androgenic actions of spironolactone are dosedependent up to at least 100-150 mg daily but prolonged daily dose of about $25 \mathrm{mg}$ of spironolactone produce generally useful anti-hypertensive effects and tolerable anti-androgen effects. However, for those requiring robust anti-androgen effects, daily doses of 150-200 mg may be necessary. This requires testing in clinical trials.

\section{Host-defense mechanisms against SARS-CoV-2 infection: angiotensin converting}

enzyme type 2 (ACE 2): A hypothesis for combined class and off target effects of spironolactone to reduce COVID-19 infectivity or disease severity is outlined in Figure 1. There has been concern that patients using a RAASi might be at increased risk for becoming infected with COVID-19 or developing organ damage, since ACE inhibitors or angiotensin receptor blockers (ARBs) can increase circulating levels of ACE2 (33), one domain of which is as the 
receptor for the binding of the spike protein of the SARS-COVID -2 virus to permit its cellular entry in the respiratory epithelium (34). However, subsequent observational studies have not reported that the use of an ACEi or ARB are not associated with an increased risk of developing or dying from COVID-19, and some have suggested beneficial effects (35). This has been ascribed to the function of the second domain of the ACE2 enzyme that metabolizes angiotensin II (Ang II) and generates Ang-1-7 that activates the Mas receptor to reduce inflammation and organ damage $(33,36)$. Spironolactone given to patients with HF increases the ACE2 levels in circulating monocytes substantially (37), although there are as yet no studies in patients with COVID-19 infection. A reduction in Ang II and an increase in Ang 1-7, may moderate the increases in reactive oxygen species and inflammation that contribute to the mortality of severe COVID-19 infection while an increase in Ang 1-7 in the lungs could counteract the effect of Ang II induced pulmonary dysfunction and fibrosis. Thus, any increase in ACE2 with spironolactone might ameliorate pulmonary injury and organ damage from COVID-19 infection. However, the complex roles of ACE2 as both an essential cell membrane receptor for SARS-CoV-2 and as a potentially protective enzyme, as discussed in great detail $(8,33)$, suggest that any benefit from increased ACE2 expression may depend on the stage and severity of the SARS-CoV-2 infection and the site of increased ACE2 expression.

Transmembrane protease receptor serine 2 (TMPRSS2): TMPRSS2, furin and plasmin are implicated in the proteolytic processing of the SARS-COV-2 spike proteins that are required for the high affinity binding to ACE2 (8). TMPRSS2 is the essential first step for proteolytic activation of SARS-COV-2 and cleaves both ACE2 and the SARS-S protein (38). It is considered a prime target to combat coronavirus infection (39). TMPRSS2 is expressed modestly in several extra pulmonary sites including human cardiomyocytes (17) where its 
expression is increased with age $(40,41)$ and male sex (42). As described (8) ACE2 and TMPRSS2 are co-expressed in cells at several strategic sites for SARS-CoV-2 infection including human and mouse conjunctiva, with increased expression in males, bronchial transient secretory cells, pulmonary type II pneumocytic cells, human nasal olfactory epithelial cells and human ileal absorptive enterocytes. Half of pulmonary transient secretory cells co-express ACE2, TMPRSS2, furin and Rho-GTPase that is a signaling molecule for aldosterone (43). Thus, strategies to reduce the expression of TMPRSS2 might reduce SARS-CoV-2 infectivity and bodily spread (8). Indeed, SARS-CoV-2 cellular entry and infectivity can be reduced by protease inhibitors such as nafamostat that inhibit TMPRSS2. This drug also is also an anticoagulant. Although the regulation of TMPRSS2 by the RAAS has yet to be studied, the gene for TMPRSS2 has an AR whose blockade reduces the lethality of SARS-CoV-2 in male mice (44). Thus, the anti-androgen action of spironolactone could reduce the expression of TMPRSS2 in cells that arecritical for SARS-CoV-2 entry and thereby reduce COVID-19 infectivity or complications (45). Therefore, the unique off-target AR blocking activity of spironolactone could be an important benefit in COVID-19 infected patients and thereby place spironolactone as the RAASi of choice for them (8). However, this will require proof from adequately powered, prospective, randomized clinical trials. While the pharmaceutical industry has focused on developing MRAs with greater specificity for the MR, the anti-androgenic of spironolactone could be a unique advantage to reduce TMPRSS2 expression and thereby reduce the risk or severity of COVID-19 infection.

Furin: As described (8), furin is a proprotein convertase that cooperates with aldosterone to enhance the activity of the epithelial sodium channel $\left(\mathrm{E}_{\mathrm{Na}} \mathrm{C}\right)$ in the renal tubular collecting ducts, pulmonary epithelium, colon, salivary glands, eye and vascular endothelium. Aldosterone 
increases furin activity (46) and regulates alveolar fluid and $\mathrm{Na}^{+}$uptake by activation of $\mathrm{E}_{\mathrm{Na}} \mathrm{C}$ where furin cooperates with aldosterone and may contribute to the ability of spironolactone to improve lung diffusion in patients with CHF (47).

Furin is expressed in human nasal epithelial cells and lung. It cleaves the S1/S2 site of the SARS-CoV-2 spike protein to enhance viral binding to ACE2 and its entry into the human lung (48). MRAs are likely to decrease furin since there is an extensive positive interaction between aldosterone and furin in renal tubules and the lung and furin activity is increased by aldosterone (46).

Plasmin: Plasmin is generated in the blood stream from plasminogen whose activity is regulated by tissue plasminogen activator (tPA) and in tissues and body fluids by urokinase (uPA). Plasmin, plasminogen and tPA/uPA generally are elevated in the body fluids of patients with the major COVID-19 risk factors (49). Plasmin may be a driver of complex dynamic interactions of hypercoagulation, leading to extensive microthrombi and organ infarction, combined with widespread anti-coagulant actions from reduced platelets, tissue hemorrhage and increased fibrinolysis termed "hemovascular dysfunction" (50). Plasminogen is expressed in the airways and the alveolar type I and II epithelial cells that are also sites of expression of ACE2 and TMPRSS2 and can cleave the spike protein of SARS-CoV-2 at the furin site, thereby enhancing binding of the virus to ACE2 and viral cell entry (51). Indeed, plasmin has been considered to be a key factor for COVID-19 susceptibility (49). Thus, plasmin and furin may cooperate with TMPRSS2 to modulate host defenses against SARS-COV-2 infection and may enhance the effects of aldosterone to activate $\mathrm{E}_{\mathrm{Na}} \mathrm{C}$ in the renal collecting ducts and pulmonary alveolae. Aldosterone increases plasmin activity (46). This suggests that spironolactone should reduce plasmin, but this has not yet been reported. 
Protease nexin 1 (PN1; serpin E2): PN1 is expressed widely, including in human nasal epithelial cells. It inhibits the activity of many proteases including $\alpha$-thrombin, plasmin, plasminogen and furin. Aldosterone reduces renal PN1 expression and thereby should increase furin and plasmin activity $(46,52)$. Thus, PN1 expression is likely to be increased by MRAs, predicting that they will reduce plasmin and furin activity and thereby reduce the proteolytic processing of the spike protein, its binding to ACE2 and virus infectivity. Inhibition of plasminogen/plasmin-induced coagulopathy could be additional benefits of an increase in PN1 with MRAs. However, the effects of MRAs on PN1 have yet to be studied directly.

Additional actions of spironolactone: Whereas a modest immune mediated inflammatory response may assist in resolving infection in patients with COVID-19 infection, some develop "hyper inflammation" or a "cytokine storm" with vasculopathy, thrombotic microangiopathy and intravascular coagulopathy that may underlie ARDS and multi-organ failure (53). The immunothrombosis can entail endothelial cell infection and dysfunction, activation of plasminogen activator inhibitor-1 (PAI-1) and platelets.

MRIs have prominent anti-inflammatory properties. Eplerenone reduces inflammation in a mouse model of viral myocarditis (54). MRAs also modulate "trained immunity", reduce inflammation and prevent vascular dysfunction in models of inflammation or hypertension (55). Thus, spironolactone could have favorable effects on the oxidative stress, hyper-inflammation and widespread coagulopathy that is reported increasingly in severe COVID-19 infections, but direct clinical trials are lacking.

Conclusion: Whereas the benefit/risk ratio of modulating ACE2 by spironolactone for prevention of COVID-19 is unclear, its "off-target "androgen receptor blocking activity is predicted to reduce TMPRSS2 and should thereby be highly protective. The activities of furin 
and plasmin are predicted to be reduced by MRAs and these effects should be amplified by PN1 whose activity is increased by MRAs. Thus, the anti-androgen actions of spironolactone may combine with its MRA activity to reduce the expression or activity of TMPRSS2, furin and plasmin and thereby reduce the high affinity binding of SARS-CoV-2 to ACE2. Additionally, the effects of MRAs to reduce ENaC activity that should be augmented by reduced furin and plasmin activity, should limit pulmonary edema, improve pulmonary gas exchange, and limit diffuse coagulation and fibrinolysis. These predictions require clinical trials to test their validity but suggest that spironolactone should protect against SARS-CoV-2 infection and also against the pulmonary, cardiac, renal and vascular inflammatory complications. Prospective clinical studies will also be required to determine the optimal dose of spironolactone and the target population. However, the anti-androgen actions of spironolactone might be enhanced by twice daily administration since it has a relatively short plasma half-life and the spironolactone molecule mediates much of the anti-androgen effects, whereas its the major metabolites mediate much of the MRA action and have a much prolonged half-life. Thus, physicians might consider using spironolactone initially twice daily in doses of 12.5 to $25 \mathrm{mg}$ for patients at high risk for SARS-COV-2 infection and in higher doses of 100-200 mg daily in those infected with the virus, providing there are no contraindications such as hyperkalemia. 
Funding: This work in CSW's laboratory was supported by the Walters Family Chair of Cardiovascular Research, the Smith-Kogod Family Foundation, the Gildenhorn-Spiesman Family Foundation and the Georgetown University Hypertension Research Center.

Acknowledgements: Not Applicable

Conflict of Interest/Disclosures: CSW has been a scientific advisor or consultant within the last year for Bristol Myers Squibb, Merck, Sanofi, Lexicon, Sarfez, PhaseBio, and Relypsa. BP is a consultant for Bayer, Astra Zeneca, Sanofi /Lexicon, Boehringer Ingelheim/Lilly, Relypsa/Vifor *, Sarfez*, SC Pharmaceuticals *, SQ innovations*, G3 Pharmaceuticals *, Cereno scientific *, Phasebio *=stock options. BP is an investor in US Patent 9931412-site specific delivery of eplerenone to the myocardium. US Patent pending 63/045,784, Histone -acetylation-modulating agents for the treatment and prevention of organ damage. 


\section{References}

1. Williams B, MacDonald TM, Morant S, Webb DJ, Sever P, McInnes G, et al. Spironolactone versus placebo, bisoprolol, and doxazosin to determine the optimal treatment for drug-resistant hypertension (PATHWAY-2): a randomised, double-blind, crossover trial. Lancet. 2015;386(10008):2059-68.

2. Pitt B, Zannad F, Remme WJ, Cody R, Castaigne A, Perez A, et al. The effect of spironolactone on morbidity and mortality in patients with severe heart failure. Randomized Aldactone Evaluation Study Investigators. N Engl J Med. 1999;341(10):709-17.

3. Pitt B, Remme W, Zannad F, Neaton J, Martinez F, Roniker B, et al. Eplerenone, a selective aldosterone blocker, in patients with left ventricular dysfunction after myocardial infarction. $\mathrm{N}$ Engl J Med. 2003;348(14):1309-21.

4. Zannad F, McMurray JJ, Krum H, van Veldhuisen DJ, Swedberg K, Shi H, et al. Eplerenone in patients with systolic heart failure and mild symptoms. N Engl J Med. 2011;364(1):11-21.

5. Cadegiani FA, Goren A, Wambier CG. Spironolactone may provide protection from SARS-CoV-2: Targeting androgens, angiotensin converting enzyme 2 (ACE2), and renin-angiotensin-aldosterone system (RAAS). Med Hypotheses. 2020;143:110112.

6. Cadegiani FA. Can spironolactone be used to prevent COVID-19-induced acute respiratory distress syndrome in patients with hypertension? Am J Physiol Endocrinol Metab. 2020;318(5):E587-e8.

7. Armanini D, Fiore C, Bielenberg J, Sabbadin C, Bordin L. Coronavirus-19: Possible Therapeutic Implications of Spironolactone and Dry Extract of Glycyrrhiza glabra L. (Licorice). Front Pharmacol. 2020;11:558418.

8. Wilcox CS, Pitt, B. Is spironolactone the preferred renin angiotensin aldosterone inhibitor for protection against covid-19? . Journal of Pharmacology. 2020; In Press.

9. Wambier CG, Goren A, Vaño-Galván S, Ramos PM, Ossimetha A, Nau G, et al. Androgen sensitivity gateway to COVID-19 disease severity. Drug Dev Res. 2020.

10. Goren A, Wambier CG, Herrera S, McCoy J, Vaño-Galván S, Gioia F, et al. Anti-androgens may protect against severe COVID-19 outcomes: results from a prospective cohort study of 77 hospitalized men. J Eur Acad Dermatol Venereol. 2020.

11. Montopoli M, Zumerle S, Vettor R, Rugge M, Zorzi M, Catapano CV, et al. Androgen-deprivation therapies for prostate cancer and risk of infection by SARS-CoV-2: a population-based study $(\mathrm{N}=4532)$. Ann Oncol. 2020;31(8):1040-5.

12. Trevisan M, de Deco P, Xu H, Evans M, Lindholm B, Bellocco R, et al. Incidence, predictors and clinical management of hyperkalaemia in new users of mineralocorticoid receptor antagonists. European journal of heart failure. 2018;20(8):1217-26.

13. Young EA, Lopez JF, Murphy-Weinberg V, Watson SJ, Akil H. The role of mineralocorticoid receptors in hypothalamic-pituitary-adrenal axis regulation in humans. The Journal of clinical endocrinology and metabolism. 1998;83(9):3339-45.

14. Struthers A, Krum H, Williams GH. A comparison of the aldosterone-blocking agents eplerenone and spironolactone. Clin Cardiol. 2008;31(4):153-8.

15. Helfer EL, Miller JL, Rose LI. Side-effects of spironolactone therapy in the hirsute woman. J Clin Endocrinol Metab. 1988;66(1):208-11.

16. Swaminathan K, Davies J, George J, Rajendra NS, Morris AD, Struthers AD. Spironolactone for poorly controlled hypertension in type 2 diabetes: conflicting effects on blood pressure, endothelial function, glycaemic control and hormonal profiles. Diabetologia. 2008;51(5):762-8.

17. Hlavacova N, Jezova D. Effect of single treatment with the antihypertensive drug eplerenone on hormone levels and anxiety-like behaviour in rats. Endocr Regul. 2008;42(4):147-53. 
18. Yang P, Shen W, Chen X, Zhu D, Xu X, Wu T, et al. Comparative efficacy and safety of mineralocorticoid receptor antagonists in heart failure: a network meta-analysis of randomized controlled trials. Heart Fail Rev. 2019;24(5):637-46.

19. Ye P, Yamashita T, Pollock DM, Sasano H, Rainey WE. Contrasting effects of eplerenone and spironolactone on adrenal cell steroidogenesis. Horm Metab Res. 2009;41(1):35-9.

20. Spironolactone and endocrine dysfunction. Ann Intern Med. 1976;85(5):630-6.

21. Huffman DH, Kampmann JP, Hignite CE, Azarnoff DL. Gynecomastia induced in normal males by spironolactone. Clin Pharmacol Ther. 1978;24(4):465-73.

22. Corvol P, Michaud A, Menard J, Freifeld M, Mahoudeau J. Antiandrogenic effect of spirolactones: mechanism of action. Endocrinology. 1975;97(1):52-8.

23. Tremblay MR, Luu-The V, Leblanc G, Noël P, Breton E, Labrie F, et al. Spironolactone-related inhibitors of type II 17beta-hydroxysteroid dehydrogenase: chemical synthesis, receptor binding affinities, and proliferative/antiproliferative activities. Bioorganic \& medicinal chemistry. 1999;7(6):1013-23.

24. Chen D, Li X, Song Q, Hu C, Su F, Dai J, et al. Assessment of Hypokalemia and Clinical Characteristics in Patients With Coronavirus Disease 2019 in Wenzhou, China. JAMA Netw Open. 2020;3(6):e2011122.

25. Liu Y, Yang Y, Zhang C, Huang F, Wang F, Yuan J, et al. Clinical and biochemical indexes from 2019-nCoV infected patients linked to viral loads and lung injury. Science China Life sciences. 2020;63(3):364-74.

26. Villard O, Morquin D, Molinari N, Raingeard I, Nagot N, Cristol JP, et al. The Plasmatic Aldosterone and C-Reactive Protein Levels, and the Severity of Covid-19: The Dyhor-19 Study. Journal of clinical medicine. 2020;9(7).

27. Henry BM, Benoit S, Lippi G, Benoit J. Circulating plasma levels of angiotensin II and aldosterone in patients with coronavirus disease 2019 (COVID-19): A preliminary report. Progress in cardiovascular diseases. 2020.

28. Hasan SS, Capstick T, Ahmed R, Kow CS, Mazhar F, Merchant HA, et al. Mortality in COVID-19 patients with acute respiratory distress syndrome and corticosteroids use: a systematic review and meta-analysis. Expert review of respiratory medicine. 2020;14(11):1149-63.

29. Dupont A. Disappearance of spironolactone-induced gynaecomastia during treatment with potassium canrenoate. Lancet. 1985;2(8457):731.

30. Francavilla A, Di Leo A, Eagon PK, Polimeno L, Guglielmi F, Fanizza G, et al. Effect of spironolactone and potassium canrenoate on cytosolic and nuclear androgen and estrogen receptors of rat liver. Gastroenterology. 1987;93(4):681-6.

31. Chapman N, Dobson J, Wilson S, Dahlöf B, Sever PS, Wedel H, et al. Effect of spironolactone on blood pressure in subjects with resistant hypertension. Hypertension. 2007;49(4):839-45.

32. Jeunemaitre X, Chatellier G, Kreft-Jais C, Charru A, DeVries C, Plouin PF, et al. Efficacy and tolerance of spironolactone in essential hypertension. Am J Cardiol. 1987;60(10):820-5.

33. Batlle D, Wysocki J, Satchell K. Soluble angiotensin-converting enzyme 2: a potential approach for coronavirus infection therapy? Clin Sci (Lond). 2020;134(5):543-5.

34. Li W, Moore MJ, Vasilieva N, Sui J, Wong SK, Berne MA, et al. Angiotensin-converting enzyme 2 is a functional receptor for the SARS coronavirus. Nature. 2003;426(6965):450-4.

35. Mehra MR, Desai SS, Kuy S, Henry TD, Patel AN. Cardiovascular Disease, Drug Therapy, and Mortality in Covid-19. N Engl J Med. 2020.

36. Touyz RM, Li H, Delles C. ACE2 the Janus-faced protein - from cardiovascular protection to severe acute respiratory syndrome-coronavirus and COVID-19. Clin Sci (Lond). 2020;134(7):747-50. 
37. Keidar S, Gamliel-Lazarovich A, Kaplan M, Pavlotzky E, Hamoud S, Hayek T, et al. Mineralocorticoid receptor blocker increases angiotensin-converting enzyme 2 activity in congestive heart failure patients. Circ Res. 2005;97(9):946-53.

38. Heurich A, Hofmann-Winkler H, Gierer S, Liepold T, Jahn O, Pöhlmann S. TMPRSS2 and ADAM17 cleave ACE2 differentially and only proteolysis by TMPRSS2 augments entry driven by the severe acute respiratory syndrome coronavirus spike protein. J Virol. 2014;88(2):1293-307.

39. Wang XD, R.; Povysil, G.; Zoghbi, A.; Motelow, J.; Hostyk, J.; Nickols, N.; Rettig, M.; Goldstein, D.B. TMPRSS2 Transcriptional Inhibition as a Therapeutic Strategy for COVID-19 Preprints 2020, 2020030360 (doi: 1020944/preprints2020030360v2). 2020.

40. Robinson EL, Alkass K, Bergmann O, Maguire JJ, Roderick HL, Davenport AP. Genes encoding ACE2, TMPRSS2 and related proteins mediating SARS-CoV-2 viral entry are upregulated with age in human cardiomyocytes. J Mol Cell Cardiol. 2020;147:88-91.

41. Dong M, Zhang J, Ma X, Tan J, Chen L, Liu S, et al. ACE2, TMPRSS2 distribution and extrapulmonary organ injury in patients with COVID-19. Biomed Pharmacother. 2020;131:110678.

42. The L. The gendered dimensions of COVID-19. Lancet. 2020;395(10231):1168.

43. Lukassen S, Chua RL, Trefzer T, Kahn NC, Schneider MA, Muley T, et al. SARS-CoV-2 receptor ACE2 and TMPRSS2 are primarily expressed in bronchial transient secretory cells. Embo j. 2020:e105114.

44. Channappanavar R, Fett C, Mack M, Ten Eyck PP, Meyerholz DK, Perlman S. Sex-Based

Differences in Susceptibility to Severe Acute Respiratory Syndrome Coronavirus Infection. J Immunol. 2017;198(10):4046-53.

45. Ghazizadeh Z, Majd H, Richter M, Samuel R, Zekavat SM, Asgharian H, et al. Androgen Regulates SARS-CoV-2 Receptor Levels and Is Associated with Severe COVID-19 Symptoms in Men. bioRxiv. 2020.

46. Kitamura K, Tomita K. Regulation of renal sodium handling through the interaction between serine proteases and serine protease inhibitors. Clin Exp Nephrol. 2010;14(5):405-10.

47. Agostoni P, Magini A, Andreini D, Contini M, Apostolo A, Bussotti M, et al. Spironolactone improves lung diffusion in chronic heart failure. Eur Heart J. 2005;26(2):159-64.

48. Hoffmann M, Kleine-Weber H, PöhImann S. A Multibasic Cleavage Site in the Spike Protein of SARS-CoV-2 Is Essential for Infection of Human Lung Cells. Mol Cell. 2020.

49. Ji HL, Zhao R, Matalon S, Matthay MA. Elevated Plasmin(ogen) as a Common Risk Factor for COVID-19 Susceptibility. Physiol Rev. 2020;100(3):1065-75.

50. Cap AP. Plasmin: a driver of hemovascular dysfunction. Blood. 2016;128(20):2375-6.

51. Kam YW, Okumura Y, Kido H, Ng LF, Bruzzone R, Altmeyer R. Cleavage of the SARS coronavirus spike glycoprotein by airway proteases enhances virus entry into human bronchial epithelial cells in vitro. PLoS One. 2009;4(11):e7870.

52. Wakida N, Kitamura K, Tuyen DG, Maekawa A, Miyoshi T, Adachi M, et al. Inhibition of prostasininduced $\mathrm{ENaC}$ activities by $\mathrm{PN}-1$ and regulation of PN-1 expression by TGF-beta1 and aldosterone. Kidney Int. 2006;70(8):1432-8.

53. Mehta P, McAuley DF, Brown M, Sanchez E, Tattersall RS, Manson JJ. COVID-19: consider cytokine storm syndromes and immunosuppression. Lancet. 2020;395(10229):1033-4.

54. Xiao J, Shimada M, Liu W, Hu D, Matsumori A. Anti-inflammatory effects of eplerenone on viral myocarditis. Eur J Heart Fail. 2009;11(4):349-53.

55. Ames MK, Atkins CE, Pitt B. The renin-angiotensin-aldosterone system and its suppression. J Vet Intern Med. 2019;33(2):363-82. 


\section{FIGURE LEGEND}

Figure 1: Hypothesis for class effects (red lettering) and off-target effects (green lettering) of spironolactone to reduce the infectivity and severity of SARS-COVID-2 infection. MRA, mineralocorticosteroid antagonist; ROS, reactive oxygen species; ACE2, angiotensin converting enzyme type 2; PN1, protein nexin 1; ENaC, epithelial sodium channel; ARDS, adult respiratory distress syndrome; TMPRSS2, transmembrane protease serine 2. 
Figure 1:

\section{HYPOTHESIS FOR CLASS-SPECIFIC AND OFF-TARGET EFFECTS OF SPIRONOLACTONE TO REDUCE THE INFECTIVITY AND SEVERITY OF SARS-COV-2 INFECTION}

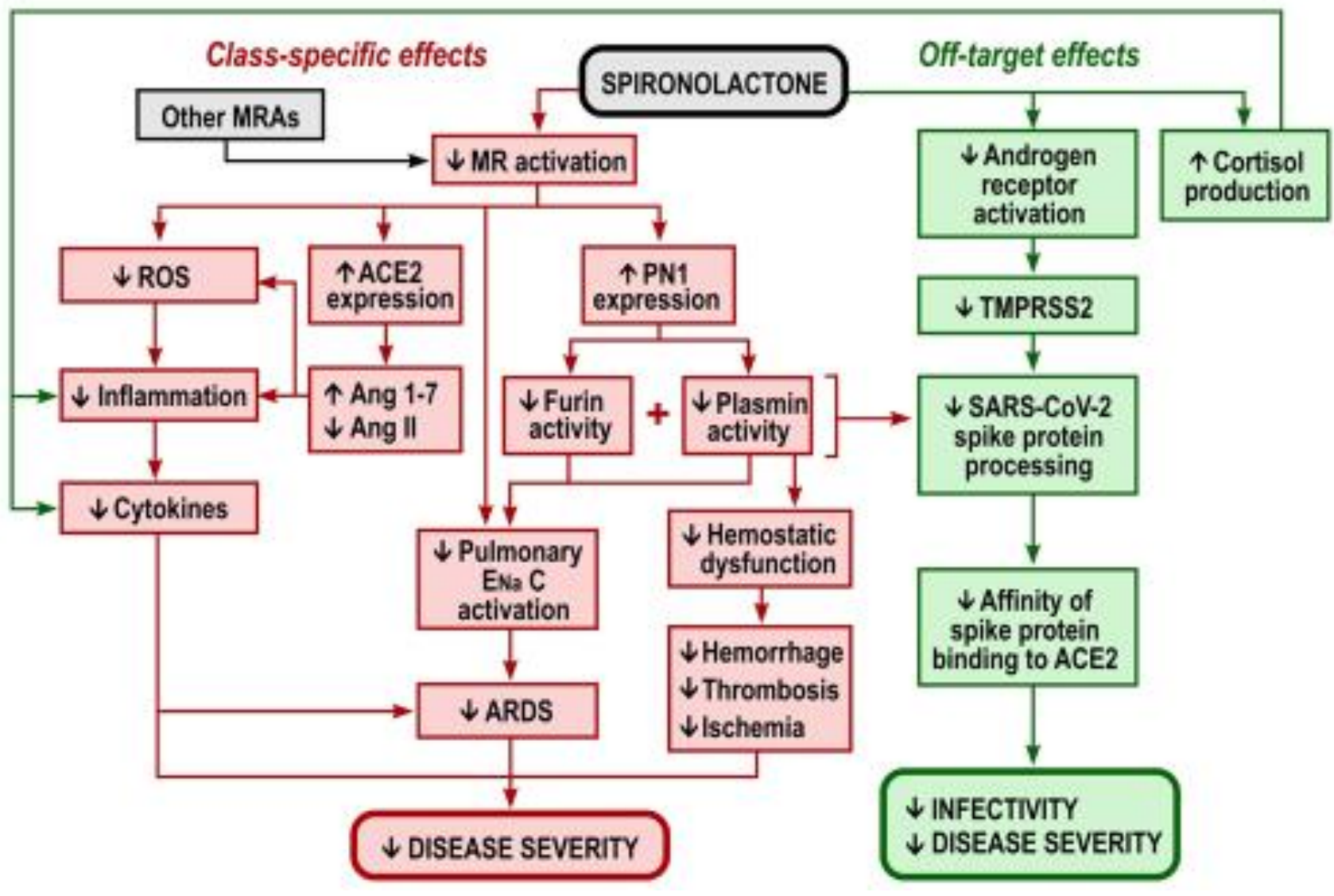

